\title{
Evaluating Ecological Vulnerability Using the GIS and Analytic Hierarchy Process (AHP) Method in Yan’an, China
}

\author{
Kang Hou, Xuxiang Li*, Jingjing Wang, Jing Zhang \\ School of Human Settlements and Civil Engineering, Xi'an Jiao tong University, \\ Xi'an, 710049, China
}

Received: 18 June 2015

Accepted: 7 January 2016

\begin{abstract}
The degraded ecosystem in the Loess Plateau region of China is very vulnerable, which has a great impact on regional sustainable development. Taking the Loess Plateau of Yan'an, Shaanxi province, China, as the study area, this study quantitatively evaluated conditions of the ecological environment. Based on natural factors, environmental factors, and socio-economic factors of Yan'an in 1997, 2004, and 2011, the dynamic changes in ecological vulnerability are analyzed with the application software for geographic information systems (GIS). The analytic hierarchy process (AHP) model was used and it contains variables for land use, soil erosion, topography, climate, vegetation, and socio-economic data. The evaluation results are divided into five categories: potential, slight, light, medium, and heavy. The results show that the ecological vulnerability in the study is light level, and the ecological vulnerability index of the southern four counties is lower than that of the northern four counties. Ecological environment quality gradually improved in most areas in 1997 2011.
\end{abstract}

Keywords: analytic hierarchy process (AHP), geographic information system (GIS), ecological vulnerability

\section{Introduction}

The Yan'an area is located in the Loess Plateau in a typical area of strong water and wind erosion. As it is a national energy and chemical base, it has important strategic significance regarding energy development. In addition, the eco-environment comprehensive evaluation is the basic premise to take effective measures to control the deterioration of the ecological environment, and effectively prevents unreasonable destruction of human

*e-mail: hupo0311@hotmail.com activities on the social ecological environment, and protects China's energy and chemical base and the safety of the Yellow River downstream. So it also provides the theoretical and scientific basis for regional ecological environmental development in an ecologically fragile area [1].

In recent years, ecological vulnerability has been developed as an important aspect of ecological assessment. The previous research studies have developed some methods, such as the fuzzy evaluation method [2, 3] and gray evaluation [4], together with the artificial neural-network evaluation method [5], and the landscape evaluation method [6]. These methods have been used 
for quantitative analysis; however, the variables used in the model are not always easily acquired and employed. For example, the neural-network method needs a number of data points from historical data, which indicates the special problem of using existing domain knowledge in the learning process $[7,8]$. The analytic hierarchy process (AHP) is a decision analysis method that considers both qualitative and quantitative information and combines them by decomposing ill-structured problems into systematic hierarchies to rank alternatives based on a number of criteria [9]. As a result, the AHP has the special advantage in multi-index evaluations in this region.

In the research of ecological environment evaluation, geographic information system (GIS) has been widely used and has become an important evaluation tool [10, 11]. The presentation of vulnerability evaluation for regional environmental protection is ideal when using GIS, in which multiple layers of information can be integrated in different combinations. This can also overcome the existing difficulties in combining numerous spatial-related parameters involved in environmental vulnerability, and thereby provide a useful and effective tool. Meanwhile, ecological models and remote sensing data will be combined through different angles, which can objectively evaluate the complex large-scale environment [12, 13]. The objective of this study was: 1) to develop a relatively reasonable regional evaluation system using GIS, 2) to quantitatively calculate a regional ecological vulnerability index using AHP, and 3) to analyze changes in the eco-

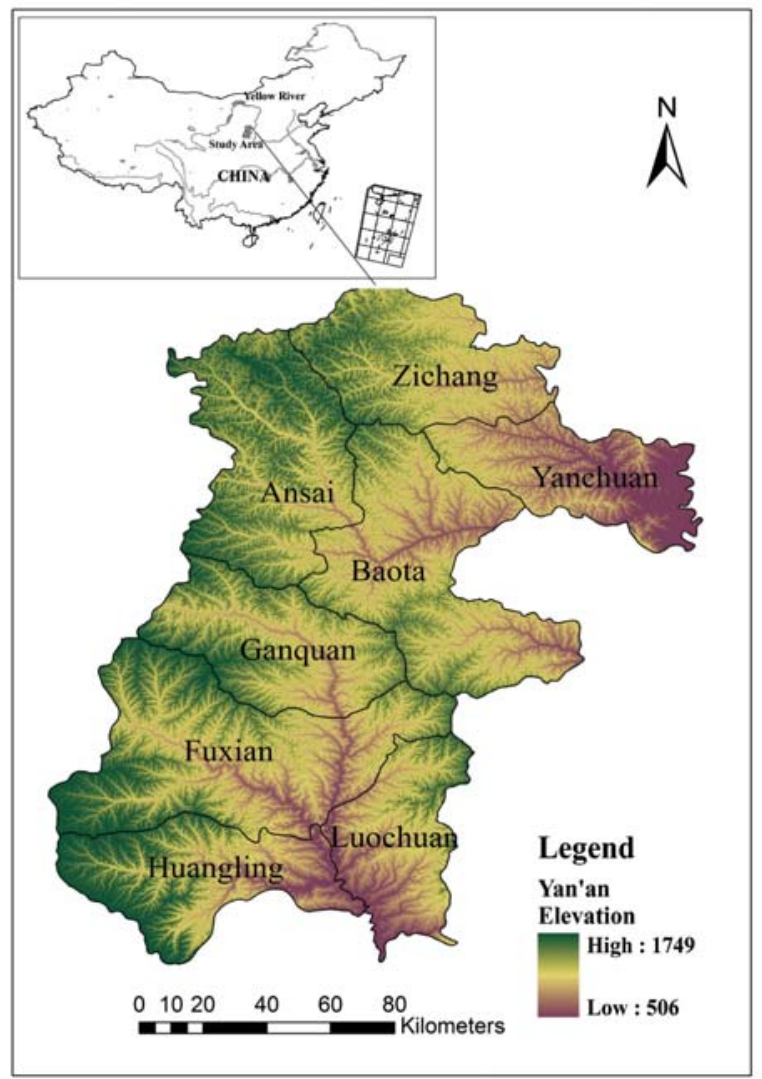

Fig. 1. Study area location. logical environmental vulnerability analysis and improve our understanding of ecological environmental changes so that a strategy of sustainable land use could be established.

\section{Study Area and Data}

The Situation of Study

The study area is located in northern Shaanxi, China, in the middle reaches of the Yellow River. It connects the four cities of Yu Lin, Wei Nan, Tong Chuan, and Xian Yang. The study area is confined by the latitudes $35.358^{\circ}$ $\sim 37.514^{\circ} \mathrm{N}$ and longitudes $108.488^{\circ} \sim 110.452^{\circ} \mathrm{E}$. The study area includes eight counties (see Fig. 1). This area is the main implementation region of the National Returning Farmland-to-Forest Project.

\section{Data}

All of the data are divided into three categories: 1) RS data is three periods of Landsat-5 TM data in 1997, 2004, and 2011, which has a spatial resolution of $30 \mathrm{~m}$. Meanwhile, in addition to remote sensing data, it also acquired the relevant data of 2) natural environment data and 3) economic and social data from the 1997, 2004, and 2011 Statistical Yearbooks.

Elevation, slope, vegetation coverage, and land use degree were interpreted from Landsat-5 resource thematic mapper (TM) images. Then, agricultural output, population density, farmland area, and per capita GDP were obtained from the Statistical Yearbook of the corresponding year (1997, 2004, and 2011 Statistical Yearbooks of Shaanxi Province and Yan'an Region). Precipitation and soil erosion were obtained from the Shaanxi Meteorological Bureau of Agricultural Remote Sensing Center.

\section{Methods}

\section{Evaluation Principles and Influence Factors}

The selection of evaluation criteria is very important in a regional environment assessment, and it should be of certain representation and maneuverability. In the quantitative study, analyzing the factors of ecological environment should consider natural factors and the impact of human activity. Various factors influencing the ecological vulnerability of the Loess Plateau are considered. But it should be noted that this selection of factors is not exhaustive, and only those salient factors for which information is of great significance were selected. Based on some previous quantitative analyses of environmental features in the study area [14], we considered all possible environmental variables for the present assessment. Fig. 2 shows an integrated evaluation criteria system. Natural factors including elevation (C1), precipitation (C), and slope (C3) form an important determinant of vulnerability evaluation [15]. Environmental factors including vegetation coverage (C4), soil erosion (C5), and 


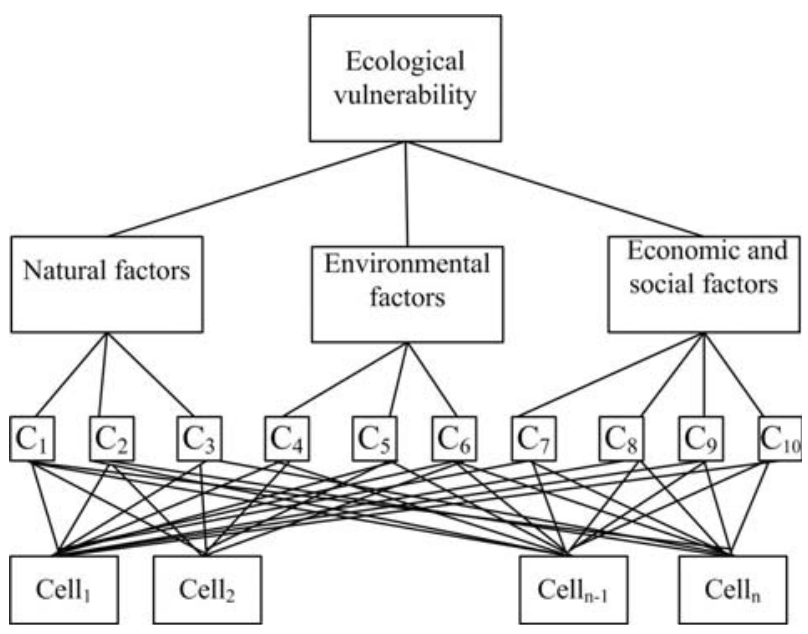

Fig. 2. An integrated evaluation criteria system in Yan'an.

land use degree (C6) form an important determinant of vulnerability evaluation [16]. The regional environmental vulnerability is also strongly related to local socioeconomic factors, since human activities can greatly influence the evolution of numerous environmental characteristics. Agricultural output (C7), per capita GDP (C8), population density (C9), and farmland area (C10) are selected to evaluate the impacts of human activities.

\section{Standardization of Factors Index}

In the process of ecological vulnerability evaluation, a primary step is to ensure a standardized measurement system for all factors considered. As a general guideline, a positive correlation between the value awarded and vulnerability is employed. All the factors were processed by this method.

$$
X=\frac{X_{i}-X_{\min }}{X_{\max }-X_{\min }}
$$

...where $i$ is the evaluation unit, $X_{\mathrm{i}}$ is the original value of $i, \mathrm{X}_{\mathrm{i} \max }$ and $\mathrm{X}_{\mathrm{i} \text { min }}$ are the maximum and the minimum value of $i$.

\section{Weight of Evaluation Factors}

The weight of each factor was determined with AHP according to the environmental conditions and expert advice. AHP was a systematic analyzing evaluation method to make the complex and multi-index system quantitatively, which could decompose the complex problem to some layers and some factors, and could compare and calculate as the result of weight. Due to its ability of assigning proper weights to various factors of complex systems, the ecoenvironmental system was suitable to employ AHP [17]. In our research, based on the Delphi expert advice system, the AHP method was applied to determine the weight of each factor [18].
In the construction of the matrix of paired comparison, the consistency of the judgments could be revealed because this matrix is a consistent matrix. For example, if factor 1 is preferred to factor 2 and factor 2 to factor 3, then factor 1 must be more preferred to factor 3. In AHP, an index of consistency, known as the consistency ratio (CR), is used to indicate the probability that the matrix judgments were randomly generated [19]:

$$
C R=\frac{C I}{R I}
$$

...where RI is the average of the resulting consistency index depending on the order of the matrix given by Saaty [20], and consistency index (CI) is defined as:

$$
C I=\frac{\lambda_{\max }-n}{n-1}
$$

$\ldots$ where $\lambda_{\text {max }}$ is the largest or principal eigenvalue of the matrix, and $n$ is the order of the matrix. A CR of 0.10 or less is a reasonable level of consistency. A CR above 0.10 requires revisions of the judgments in the matrix because of an inconsistent treatment of particular factor ratings. In this study, the maximum CR of 0.04 is then acceptable. Eventually, all the factors weigh as shown in Table 1.

\section{Environmental Vulnerability Index} (EVI)

The higher the EVI value, the more vulnerable the ecological environment is. Using the weighted linear combinations method, the evaluation model is built to calculate the EVI. The factors evaluated were combined by applying a weight of each factor, followed by a summation of the results to yield a vulnerability index. The evaluation value of each unit was the sum of the

\begin{tabular}{|c|c|c|c|c|}
\hline $\begin{array}{l}\text { First } \\
\text { grade }\end{array}$ & $\begin{array}{l}\text { Second } \\
\text { grade }\end{array}$ & Weight & Third grade & Weight \\
\hline \multirow{10}{*}{ A } & \multirow{3}{*}{ B1 } & \multirow{3}{*}{0.1958} & $\mathrm{C} 1$ & 0.0172 \\
\hline & & & $\mathrm{C} 2$ & 0.1311 \\
\hline & & & $\mathrm{C} 3$ & 0.0475 \\
\hline & \multirow{3}{*}{ B2 } & \multirow{3}{*}{0.3108} & $\mathrm{C} 4$ & 0.0741 \\
\hline & & & $\mathrm{C} 5$ & 0.1943 \\
\hline & & & $\mathrm{C} 6$ & 0.0424 \\
\hline & \multirow{4}{*}{ B3 } & \multirow{4}{*}{0.4934} & $\mathrm{C} 7$ & 0.0375 \\
\hline & & & $\mathrm{C} 8$ & 0.1333 \\
\hline & & & $\mathrm{C} 9$ & 0.2624 \\
\hline & & & $\mathrm{C} 10$ & 0.0602 \\
\hline
\end{tabular}

Table 1. Relative weights of factors for environmental vulnerability evaluation. 
Table 2. Ecological vulnerability classification in Yan'an.

\begin{tabular}{|c|c|c|}
\hline Evaluation level & EVI & Feature description \\
\hline Potential & 0.80 & Stable ecosystem, super high antijamming capability, rich soil, and good vegetation cover \\
\hline Slight & $0.80 \sim 1.01$ & Relatively stable ecosystem, antijamming capability, rich soil, and relatively good vegetation \\
cover
\end{tabular}

corresponding weight values of all selected factors. So the ecological vulnerability index can be calculated by following formula:

$$
E V I=\sum_{i=1}^{10} w_{i} f_{i}
$$

...where $E V I$ is the environmental vulnerability index, $w_{i}$ the weight of factor $i$, and $f_{i}$ the rating of factor $i$.

\section{The Classification of Ecological Vulnerability Index}

The EVI was could be classified into several ranks to reflect the different vulnerability levels. The natural breaks classification (NBC) is an objective and rational measure to analyze the statistical distribution in the attribute space. It can identify break points by picking the class breaks that group similar values and maximize the differences between classes, and the features are divided into classes whose boundaries are set where there are relatively large jumps in the data values [16]. In this study, the method of the natural breaks classification was used to divide the ecological vulnerability assessment into five grades, and each grade was presented in Table 2.

\section{Results and Discussion}

Calculating Ecological Vulnerability Index and Mapping the Ecological Vulnerability Index Chart in 1997, 2004, and 2011

Using these formulas, the ecological vulnerability index of the Yan'an area can be calculated in 1997, 2004, and 2011. Based on ecological vulnerability data in the study area, the Natural Breaks Classification method was divided into five grades: potential, slight, light, medium, and heavy.

According to factor weights and the ecological vulnerability classification standard (Tables 1 and 2), the spatial multi-temporal assessments of the ecological vulnerability index that are classified in 1997, 2004, and 2011 are shown in Fig. 3 and Table 3. In 1997, the light vulnerable zone with the largest area proportion accounting for $29.86 \%$, the medium vulnerable zone accounts for $29.42 \%$, the heavy vulnerable zone accounts for $20.27 \%$, the slight vulnerable zone accounts for $16.98 \%$, and the potential vulnerable zone only accounts for $3.47 \%$. In 2000 , the light vulnerable zone with the largest area proportion accounted for $30.14 \%$, the medium vulnerable zone for $28.43 \%$, the slight vulnerable zone $24.17 \%$, the heavy vulnerable zone $12.71 \%$, and the potential vulnerable zone only for $4.55 \%$. In 2011, the light vulnerable zone with the largest area proportion accounts for $30.81 \%$, the medium vulnerable zone for $29.31 \%$, the slight vulnerable zone for $20.73 \%$, the heavy vulnerable zone for $15.11 \%$, and the potential vulnerable zone only for $3.98 \%$.

\section{Changes in the Ecological Vulnerability Index in Administrative Region}

In 1997-2011, the ecological vulnerability index decreased in Yan'an, which means that the ecological environment was gradually stabilizing. However, in some counties the ecological vulnerability index gradually increased. The ecological vulnerability indexes of Baota District, Ansai County and Zichang County are abnormal, where the EVI of the latter stage is larger than the earlier stage. The EVI in four southern counties gradually became smaller from 1997 to 2011, and therefore the security of the ecological environment has gradually increased.

In the spatial scale, most counties show a clearly horizontal distribution (see Fig. 3). Among them, the EVI of four northern counties (including Baota District, Zichang County, Ansai County, and Yanchuan County) was significantly higher than the four southern counties (including Ganquan, Fuxian, Hanging, and Luochuan counties). In other words, environmental safety from north to south gradually becomes stronger, and then such incremental changes are closely related to rainfall and human economic development.

The ecological vulnerability indexes of Ganquan County and Fu County are the lowest, which can also illustrate regional ecological environmental security and stability. In eight areas, the ecological vulnerability of Luochuan County and Baota District is higher than other counties, where urban expansion is one of the most important factors. 

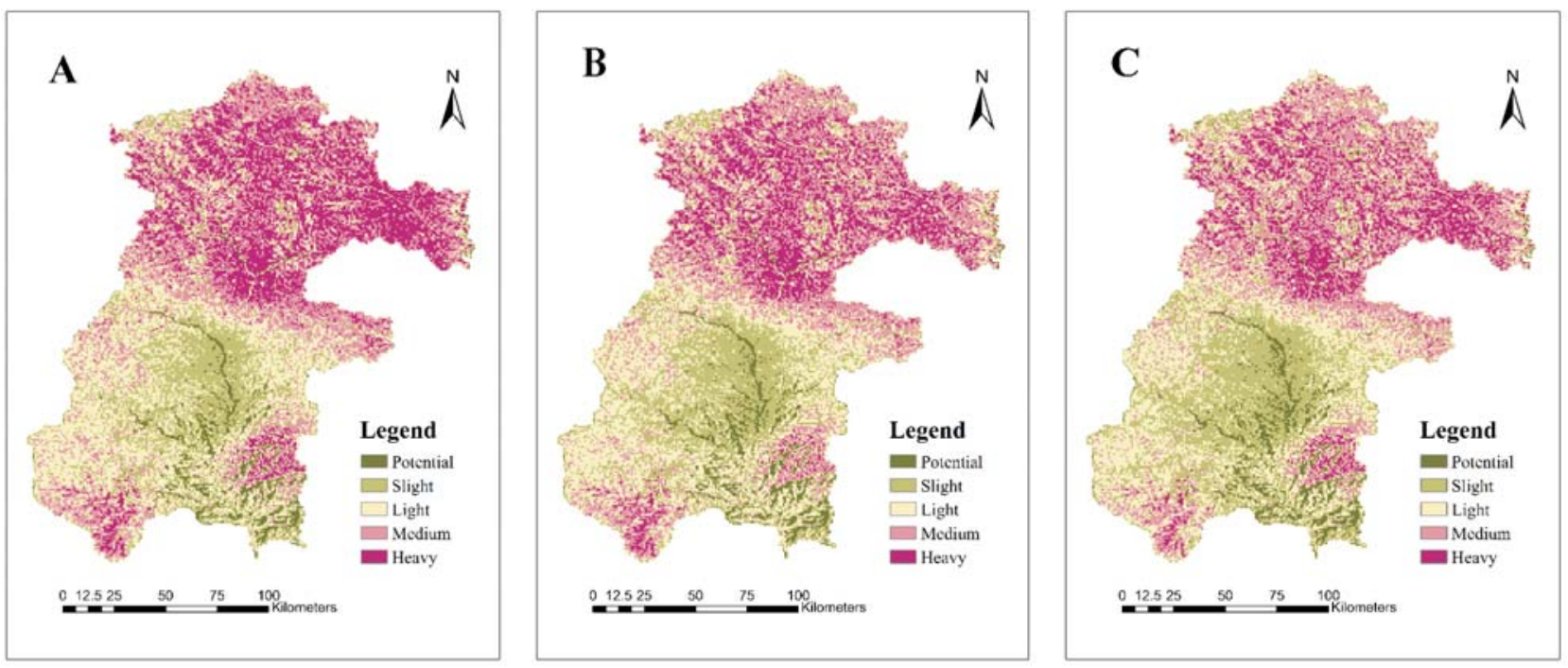

Fig. 3. The ecological environmental vulnerability of Yan'an in 1997(A), 2004(B), and 2011(C).

\section{Ecological Vulnerability Changes over 15 Years}

In 1997-2011, the ecological vulnerability index decreased for the study area, and the ecological environment gradually stabilized. In 1997-2004 and $2004-2011$, the ecological vulnerability indices of Baota, Ansari, and Zichang counties were abnormal because the later ecological vulnerability index was larger than for the earlier period. Among these counties, the ecological vulnerability index of four northern counties (including Baota District and Ansai, Zichang, and Yanchuan counties) was significantly higher than that of four southern counties (include Fu xian, Ganquan, Huangling, and Luochuan). In other words, the safety of the ecological environment going north to south gradually became stronger.

\section{Effect of the Main Driving Factors}

From 1997 to 2011, except for a few places, ecological vulnerability index gradually decreased in most of the areas, which reflected that the ecological environmental quality had been improved. Many factors can affect the ecological environmental stability. In general, the economic development and vegetation cover were the main driving forces in affecting ecological environmental vulnerability.

Table 3. The results of proportion of each level in the study area.

\begin{tabular}{|c|c|c|c|}
\hline EVI Grade & $1997 \mathrm{EVI}$ & $2004 \mathrm{EVI}$ & $2011 \mathrm{EVI}$ \\
\hline Potential & $3.47 \%$ & $4.55 \%$ & $3.98 \%$ \\
\hline Slight & $16.98 \%$ & $24.17 \%$ & $20.73 \%$ \\
\hline Light & $29.86 \%$ & $30.14 \%$ & $30.81 \%$ \\
\hline Medium & $29.42 \%$ & $28.43 \%$ & $29.31 \%$ \\
\hline Heavy & $20.27 \%$ & $12.71 \%$ & $15.17 \%$ \\
\hline
\end{tabular}

The influence of economic development is primarily divided into two categories: energy exploitation and urbanization development. The Yan'an area is the National Energy Chemical Base, and economic development mainly depends on energy development. Energy exploitation directly causes soil erosion in fragile ecological environmental areas and it can directly affect the sustainable development of the ecological environment. In recent years, the Chinese government has paid more attention to the energy exploitation control and ecological environmental sustainable development in Loess Plateau of Yan'an. More sloping fields have been converted to grassland and woodland in the past decade $[21,22]$. In addition, the rapid development of urbanization has an effect on land use, and then it increases the demand for resources.

In the ecologically vulnerable areas of Loess Plateau, vegetation is an important factor affecting the ecological environmental balance. Because of the serious soil and water erosion, the vegetation has been degraded, then mining of coal and oil also seriously have damaged the growth of vegetation. Because of the limitations of the natural environment, the effect of returning farmland to forests is not so obvious in some places, such as Ansai County. Therefore, a reasonable vegetation restoration program is the focus of future work as it directly affects the degree of ecological environmental vulnerability.

\section{Sustainable Development of Ecological Environment}

Yan'an has the complex topography of a loess region and fragile ecological environment. In the Loess Plateau, $60 \%$ of the land has a greater than 25 degree slope, which is not appropriate for growing crops. The strong gravitational erosion in the loess slope zone can lead to geological hazards. Meanwhile, the region's energy and chemical industries support the development of the 
economy, and exploitation of oil and gas resources and coal mining has a sizeable effect on land use. The forest coverage rate was rather low in economically developed districts, such as the northern counties, but the ecological vulnerability index was higher than economically developing districts in southern counties. From the view of regional development, economically developed locations also have similar laws, which show that the stability of the region's economic development is closely related to the environment.

Yan'an is part of the National Energy and Chemical Base, the ecological security in Yan'an area cannot be ignored. The local government should therefore follow the laws of nature, reduce the interference of human activities on the environment, and achieve sustainable development of the ecological environment in energy-based urban regions.

\section{Conclusions}

This study focuses on the ecological vulnerability in a typical zone: the Yan'an area in the loess plateau. Moreover, it quantitatively analyzed changes of ecological vulnerability using GIS software and the AHP method.

The evaluation of ecological vulnerability for Yan'an showed that regional areas of potential, slight and light grade approximated to $3 / 5$ of the total area, and those areas gradually increased from 1997 to 2011. This result indicated that the ecological environment became more stabilized. In addition, the ecological vulnerability index of the four northern counties is higher than that of the four southern counties.

Because of the limits of meteorological data sources and the number of monitoring sites, it was difficult to completely present the regional internal changes in the ecological environment. Therefore, evaluation accuracy could be enhanced by collecting more detailed data to strengthen regional evaluation research.

\section{Acknowledgements}

We would like to thank the anonymous reviewers for their constructive comments on earlier versions of the manuscript. The original manuscript was revised by American Journal Experts (AJE). We also are thankful to the institution that provided accurate data.

\section{References}

1. ZHANG K.M., WEN Z.G. Review and challenges of policies of environmental protection and sustainable development in China. Journal of environmental management. 88 (4), 1249, 2008.

2. JANSSEN J.A.E.B., KROL M.S., SCHIELEN R.M.J., HOEKSTRA A.Y., DE KOK J.L. Assessment of uncertainties in expert knowledge, illustrated in fuzzy rule-based models. Ecological Modelling. 221 (9), 1245, 2010.
3. LIU K.F.R., LAI J.H. Decision-support for environmental impact assessment: A hybrid approach using fuzzy logic and fuzzy analytic network process. Expert Systems with Applications. 36 (3), 5119, 2009.

4. XIAODAN W., XIANGHAO Z., PAN G. A GIS-based decision support system for regional eco-security assessment and its application on the Tibetan Plateau. Journal of environmental management. 91 (10), 1981, 2010.

5. BESAW L.E., RIZZO D.M., KLINE M., UNDERWOOD K.L., DORIS J.J., MORRISSEY L.A., PELLETIER K. Stream classification using hierarchical artificial neural networks: A fluvial hazard management tool. Journal of hydrology. 373 (1), 34, 2009

6. M MENZEL S., NORDSTRÖM E.M., BUCHECKER M., MARQUES A., SAARIKOSKI H., KANGAS A. Decision support systems in forest management: requirements from a participatory planning perspective. European Journal of Forest Research. 131 (5), 1367, 2012.

7. LUO J., WEI Y.H.D. Modeling spatial variations of urban growth patterns in Chinese cities: the case of Nanjing. Landscape and Urban Planning. 91 (2), 51, 2009.

8. NETO A.C.L., LEGEY L.F.L., GONZÁLEZ-ARAYA M.C., JABLONSKI S. A system dynamics model for the environmental management of the Sepetiba Bay watershed, Brazil. Environmental management. 38 (5), 879 , 2006.

9. CHEN M.F., TZENG G.H., DING C.G. Combining fuzzy AHP with MDS in identifying the preference similarity of alternatives. Appl. Soft Comput. 8, 110, 2008.

10. INGLADA J., MERCIER G. A new statistical similarity measure for change detection in multitemporal SAR images and its extension to multiscale change analysis. Geoscience and Remote Sensing, IEEE Transactions on. 45 (5), 1432, 2007.

11. GARNIER E., NAVAS M.L. A trait-based approach to comparative functional plant ecology: concepts, methods and applications for agroecology. A review. Agronomy for Sustainable Development. 32 (2), 365, 2012.

12. LE Q.B., PARK S.J., VLEK P.L., CREMERS A.B. Land-Use Dynamic Simulator (LUDAS): A multi-agent system model for simulating spatio-temporal dynamics of coupled humanlandscape system.I. Structure and theoretical specification. Ecological Informatics. 3 (2), 135, 2008.

13. LI X., DAMEN M.C.J. Coastline change detection with satellite remote sensing for environmental management of the Pearl River Estuary, China. Journal of Marine Systems. 82, S54, 2010.

14. LIU L., LIU X.H. Sensitivity analysis of soil erosion in the northern Loess Plateau. Procedia environmental sciences. 2, 134, 2010.

15. HUANG I.B., KEISLER J., LINKOV I. Multi-criteria decision analysis in environmental sciences: ten years of applications and trends. Science of the total environment. 409 (19), $3578,2011$.

16. LI A., WANG A., LIANG S., ZHOU W. Eco-environmental vulnerability evaluation in mountainous region using remote sensing and GIS - a case study in the upper reaches of Minjiang River, China. Ecological Modeling. 192, (1), 175, 2006.

17. LI Z.W., ZENG G.M., ZHANG H. The Integrated Eco-environment Assessment of the Red Soil Hilly Region Based on GIS - A Case Study in Changsha City, China. Ecol. Model. 202 (3-4), 540, 2007

18. SIPAHI S., TIMOR M. The analytic hierarchy process and analytic network process: an overview of applications. Management Decision. 48 (5), 775, 2010. 
19. XIAODAN W., XIANGHAO Z., PAN G. A GIS-based decision support system for regional eco-security assessment and its application on the Tibetan Plateau. Journal of environmental management. 91 (10), 1981, 2010.

20. ISHIZAKA A., LABIB A. Analytic hierarchy process and expert choice: Benefits and limitations. OR Insight. 22, (4), 201, 2009.
21. DENG J.S., WANG K., HONG Y., QI J.G. Spatio-temporal dynamics and evolution of land use change and landscape pattern in response to rapid urbanization. Landscape and Urban Planning. 92 (3) 187, 2009.

22. WANG S.Y., LIU J.S., MA T.B. Dynamics and changes in spatial patterns of land use in Yellow River Basin, China. Land Use Policy. 27 (2), 313, 2010. 
